\title{
Laser interaction with materials: introduction
}

\author{
Claude R. Phipps, ${ }^{1, *}$ (1) Leonid Zhigilei, ${ }^{2}$ Pavel Polynkin, ${ }^{3}$ Vitaly Gruzdev, ${ }_{9}^{4}$ Willy Bohn, ${ }^{5}$ \\ and Stefan Scharring ${ }^{6}$ \\ ${ }^{1}$ Photonic Associates, LLC, 200A Ojo de la Vaca Road, Santa Fe, New Mexico 87508, USA \\ ${ }^{2}$ Department of Materials Science and Engineering, University of Virginia, 395 McCormick Road, Charlottesville, Virginia 22904-4745, USA \\ ${ }^{3}$ College of Optical Sciences, University of Arizona, 1630 East University Blvd., Tucson, Arizona 85721, USA \\ ${ }^{4}$ Department of Physics and Astronomy, University of New Mexico, 1919 Lomas Blvd., Albuquerque, New Mexico 87131, USA \\ ${ }^{5}$ BohnLaser Consult, Weinbergweg 43, 70569 Stuttgart, Germany \\ ${ }^{6}$ German Aerospace Center (DLR), Institute of Technical Physics, Pfaffenwaldring 38-40, 70569 Stuttgart, Germany \\ *Corresponding author: crphipps@aol.com
}

Received 24 September 2018; posted 24 September 2018 (Doc. ID 346716); published 1 October 2018

\begin{abstract}
Laser-material interaction is the fascinating nexus where laser physics, optical physics, and materials science intersect. Applications span the range from high-intensity shock physics at the nanoscale to futuristic ideas like space propulsion using remotely located lasers. These papers were selected from the program of the 2018 International High Power Laser Ablation Symposium in Santa Fe, New Mexico, held 26-29 March 2018, or contributed later by our attendees. This conference was held on the 20th anniversary of our first meeting in 1998. () 2018 Optical Society of America
\end{abstract}

https://doi.org/10.1364/JOSAB.35.00LIM1

Topics of papers in this feature issue were specially selected from those submitted to or presented at the International High Power Laser Ablation Symposium held in Santa Fe, 26-29 March 2018.

This meeting was the 20th anniversary of the International High Power Laser Ablation Symposia (HPLA). HPLA meetings have provided a unique forum for exchange of ideas on the physics and application of high-power laser-material interaction, including advances in relevant high-power laser sources and problems of beam propagation and detection, in a collegial atmosphere. At its formation, the HPLA series was one of the first to be organized around a broad physical application (laser ablation) rather than a single technology. We named it HPLA to distinguish it from COLA, HPLS\&A, and other meetings in this field.

This special JOSA B issue is aimed at scientists and engineers interested in understanding how pulsed lasers interact with different materials, producing new states of matter, or new capabilities that cannot be had any other way. For example, rocket exhaust velocity $v_{E}$ produced by chemistry is limited to about $5 \mathrm{~km} / \mathrm{s}$, but laser ablation jet temperatures can easily give $20-30 \mathrm{~km} / \mathrm{s}$. This is important because payload mass that can be delivered to space increases with $v_{E}^{2}$. In turn, this corresponds to a deliverable mass ratio increase for a space mission from present-day $5 \%$ to $50 \%$, and this dramatically reduces launch cost.

Another example: materials at high pressure tend to be opaque. Hence, for their analysis, extremely thin samples must be studied in order to get information about time-dependent chemistry and optical properties of the materials. With high-power ultrashort pulses, we can obtain micrometer-scale shocked regions and use the beam that created them to probe the material and get this information.

Numerous disciplines are reflected in the work presented here. These include laser physics, optical physics, optical engineering, nonequilibrium chemistry at states of temperature and pressure not normally achieved on Earth, nanoengineering, plasma dynamics, semiconductor physics, shock physics, simulation, and modeling.

\section{Highlights of This Issue}

D. S. Moore (Los Alamos National Lab) provides an indepth review of shock-induced chemistry and phase transitions at the nanoscale. At these scales, nonequilibrium material dynamics evolve. Measurements under these conditions used to be attempted using laser-accelerated foils ("flyer plates") colliding with the test object, a brute-force method compared to what can be produced in the material with ultrashort pulses. The paper shows us how to generate nanoscale shocks as well as how to obtain data from these shocks with nanometer special scale at sub-ps times while avoiding flyer plates altogether. The author used a temporal pulse shaping method that achieves a very fast leading edge and a region of fairly constant intensity. With $5-8 \mathrm{~km} / \mathrm{s}$ shock velocity $(5-8 \mathrm{~nm} / \mathrm{ps})$, nm-scale target surface uniformity was required. The author discusses how to achieve this, as well as the methods of frequency domain interferometry and ultrafast dynamic ellipsometry necessary to do the associated diagostics.

Bauer and Perram (Air Force Institute of Technology, Wright-Patterson Air Force Base, Ohio) report highly nonMaxwellian velocity distributions in laser-ablated titanium 
vapor expanding into helium and argon. When flow speeds are larger than $0.1 \mathrm{~cm} / \mu \mathrm{s}$, multimodal, shifted MaxwellBoltzmann velocity distributions are appropriate only for expansion into vacuum. Armed with $\mathrm{Ti}$ ion time-of-flight data with high spatial resolution, the authors saw three distinct distributions, the fastest of these coming from a front of ionized $\mathrm{Ti}$ moving at $16 \mathrm{~km} / \mathrm{s}$. The plasma plumes studied were initiated by $25 \mathrm{~ns}$ pulses of $\mathrm{KrF}$ laser radiation, and $512 \times 512$ element images were captured with a gated ICCD camera. An extended discussion of the conversion of this data into multimodal velocity distributions is provided.

Kudryashov et al. (ITMO University, St. Petersburg) found they could study multiphoton absorption in fused BK-7 silica glass by carefully studying the dimensions of craters produced in ablation of the material by $4 \mu \mathrm{J}, 550 \mathrm{~nm}, 220 \mathrm{fs}$ pulses at $0-2 \mathrm{MHz}$ repetition rate. The dependence of crater diameter on single-pulse energy could be explained by assuming one-, two-, and four-photon absorption processes. The depth profiles were considered as sets of distances representing nonlinear transmission of the incident Gaussian beams until the ablation fluence level is reached, and analyzed in terms of multiphoton absorption mechanisms. With these short pulses, plasma was absent during the interaction and did not need to be considered.

Milov et al. (University of Twente) report a computational study of damage in thin ruthenium films due to XUV pulses. A combined Monte Carlo-two-temperature model was used. These are of interest because of the possible use of ruthenium as grazing incidence mirrors for XUV and x-ray beams. One specific model considered is $200 \mathrm{~nm}$ thick Ru film at $92 \mathrm{eV}$ photon energy and $20^{\circ}$ grazing incidence angle, where a surface reflectivity of $68 \%$ is calculated for incident fluence of $200 \mathrm{~mJ} / \mathrm{cm}^{2}$. Other situations are also modeled in the paper. Electron-phonon coupling, electron specific heat, photon attenuation length, and other parameters are included in deriving the results.

Van Woerkom et al. (Air Force Institute of Technology) used an Er:YAG laser with $60 \mu$ s pulses to study he emission spectra of $\mathrm{AlO}$ from ablated aluminum. Incident fluence was about $250 \mathrm{~J} / \mathrm{cm}^{2}$. A temperature of about $3000 \mathrm{~K}$ was deduced. A rovibronic model including self-absorption within the plume was used to determine the results. A method was developed to rapidly analyze hundreds of spectra taken as the background pressure was varied from 400 to 1000 mbar. It was found that the $\mathrm{AlO}$ temperature was substantially independent of background pressure. Because the observed spectra were necessarily spatially and temporally averaged, while the ejected plume is spatially inhomogeneous and evolves in time, direct conclusions about the AlO chemical kinetics could not be made. However, semiquantitative comparisons with other ablation studies were made.

Polkynkin et al. (University of Arizona) report on a new technique for laser machining using spatially and temporally focused (SSTF) femtosecond vortex beams. Experiments were conducted on $150 \mu \mathrm{m}$ thick borosilicate glass. The back side of the glass sample was machined. The goal was to eliminate a sort of "Bailey's Beads" result on the back surface as self-focusing on passing through the sample produced filamentation of the desired annular pattern. The input beam was positively chirped to compensate for the negative chirp introduced by the gratings in the SSTF setup. The desired result was obtained using both single-shot and multishot illumination. In a general sense, their results show an interesting way to avoid filamentation due to nonlinear refraction in glass.

Sergaeva et al. (University of Missouri) report simulations of ultrafast excitation of conduction band electrons using intense, mid-infrared femtosecond pulses. Novel physics is found for the free-carrier heating. The Keldysh-Vinogadov model was applied to $\mathrm{ZnSe}$, and a comparison made to results of the Drude model. It was found that the Drude model significantly overestimates free-carrier heating, with one order of magnitude higher free-carrier energy.

Phipps et al. (Photonic Associates, Santa Fe) report a detailed plan for using laser ablation of a shell surrounding a $10-13 \mathrm{~kg}$ satellite payload to launch large numbers of such objects into low Earth orbit (LEO), into geosynchronous orbit (GEO), or into a Hohmann transfer orbit to Mars. They consider a $100 \mathrm{~kW}$ average power $355 \mathrm{~nm}$ laser with 100 ps pulses. The goal was to avoid less practical $1 \mathrm{MW}$ lasers considered in earlier publications. This laser is considered possible with modest development of existing laser technology. Ablation propulsion relationships are provided, including energetic efficiency, fuel lifetime, and optimum coupling coefficient (thrust per watt) to obtain these trajectories. Drift of RAAN and periapsis due to Earth's oblateness are included. 